the development of sexual health interventions, we have long recognised that engagement with individuals and communities is a critical part of the research process, and actively sought out opportunities to collaborate with non-academic partners with a view to ensuring that our work is relevant in the 'real-world'. While the increasing call for 'collaboration', 'patient and public involvement' and 'co-production' is welcome, we recognise the possibility that without critical reflection on the part of the research team, such engagement can lack meaning for those being asked to participate, potentially reducing its value and risking it being perceived as tokenistic. In this paper we reflect on our experiences as qualitative researchers for whom a key role in interdisciplinary research teams has been undertaking a number of 'patient public involvement' projects with both young people and gay, bisexual and other men who have sex with men (MSM) to inform the development of sexual health interventions. We critically reflect on some of the challenges and opportunities involved in this form of 'consultation', and explore the ethical implications of engaging stakeholders in identifying issues they consider important as part of the design and delivery of future interventions, but which may not be considered either a priority or feasible.

\section{PP MEANINGFUL AND ENGAGING, OR TOKENISTIC? REFLECTIONS ON COLLABORATIVE ENGAGEMENT IN THE PROCESS OF DESIGNING SEXUAL HEALTH INTERVENTIONS}

N Boydell, ${ }^{*}$ L McDaid. MRC/CSO Social and Public Health Sciences Unit, University of Glasgow, UK

\subsection{6/bmjopen-2017-016492.42}

Working with non-academic partners and including participants, and other stakeholders, in the design and delivery of research is emerging as a critical element in the field of health research. As researchers based in an interdisciplinary team with a focus on 